\title{
Supratentorial Hemangioblastoma with Unusual Features
}

\author{
Yooju Shin · Seokhwi Kim · Hyun-Woo Lee · Heejin Bang • Yeon-Lim Suh \\ Department of Pathology and Translational Genomics, Samsung Medical Center, Sungkyunkwan University School of Medicine, Seoul, Korea
}

Hemangioblastomas of the central nervous system (CNS) are benign tumors that most commonly occur in the cerebellum, followed by the brainstem and spinal cord. ${ }^{1}$ Supratentorial location is extremely rare. ${ }^{2} \mathrm{CNS}$ hemangioblastomas can occur sporadically or in association with von Hippel-Lindau disease. ${ }^{2}$ Histologically, the tumors are composed of two main components: large and vacuolated stromal cells and numerous thinwalled vessels. Due to the characteristic lipid-containing cytoplasm and foci of pleomorphic nuclei, the differential diagnosis of hemangioblastoma from some other malignant tumors, such as clear cell renal cell carcinoma (RCC), adrenal cortical carcinoma and paraganglioma, remains challenging, especially in cellular variants. Hyaline globules can be found in many different tumors and benign tissues, ${ }^{3}$ including CNS hemangioblastomas, and they contribute to the diagnostic difficulty. Herein, we report a case of sporadic supratentorial hemangioblastoma with unusual features and discuss the differential diagnosis of CNS hemangioblastoma.

\section{CASE REPORT}

A previously healthy 48-year-old female had developed weakness on the right side and clumsiness in the hands three weeks before admission. At an outside hospital, brain magnetic resonance imaging revealed a heterogeneously enhancing left frontal lobe mass measuring $3.3 \times 2.6 \mathrm{~cm}$ (Fig. 1). The mass con-

\section{Corresponding Author}

Yeon-Lim Suh, M.D.

Department of Pathology and Translational Genomics, Samsung Medical Center,

Sungkyunkwan University School of Medicine, 81 Irwon-ro, Gangnam-gu,

Seoul 135-710, Korea

Tel: +82-2-3410-2800, Fax: +82-2-3410-0025, E-mail: ylsuh76@skku.edu

Received: June 25, 2014 Revised: August 28, 2014

Accepted: September 1, 2014 tained a cystic or necrotic component, and the radiologic differential diagnosis included metastasis and high-grade glioma. A craniotomy was performed, and the tumor was resected. During surgery, abnormal vascular structures both inside and around the tumor were found. Upon gross examination, the tumor was a soft and friable yellow mass.

Microscopically, the tumor was well delineated from the surrounding brain parenchyma and characterized by an abundant arborizing capillary network and sheets of pleomorphic polygonal cells (Fig. 2A). The nuclear morphology of the tumor cells was variable: single or multiple (up to 10 ), round to elongated, dark or vesicular, pleomorphic or bizarre nuclei with often multiple or single prominent nucleoli, and frequent intranuclear cytoplasmic inclusions. The cytoplasm was abundant and eosinophilic or clear and occasionally uni- or multi-vacuolated (Fig. 2B). Tumor cells with eccentrically located nuclei and glassy cytoplasm were also identified. Neither necrosis nor mitotic activity was seen. Numerous extracellular and intracytoplasmic hyaline globules were observed (Fig. 2C). The presence of pleomorphic, vacuolated clear cells and hyaline globules raised the suspicion of metastatic tumors including RCC, adrenal cortical carcinoma and hemangioblastoma.

Immunohistochemical staining was done using the Leica BondMax automatic stainer. Antibodies used included the markers of RCC (EMA, 1:300, Dako, Glostrup, Denmark; pancytokeratin, 1:500, Dako; vimentin, 1:2,000, Dako; CD10, 1:250, Novocastra, Newcastle upon Tyne, UK; PAX2, 1:100, Invitrogen, Carlsbad, CA, USA; CA IX, 1:1,000, Abcam, Burlinggame, CA, USA), of adrenal tumors ( $\alpha$-inhibin, 1:40, Serotec, Oxford, UK; Melan-A, 1:80, Dako) and of hemangioblastoma (D2-40, 1:100, Dako). The tumor cells diffusely expressed vimentin and CD10, and they focally expressed S-100, $\alpha$-inhibin, CA IX, and D2-40 (Fig. 3). Melan-A and EMA-positive cells were identi- 
fied. AE1/AE (1:500, Dako), glial fibrillary acidic protein (1:400, Novocastra), synaptophysin (1:100, Dako), and chromogranin (1:400, Dako) were negative. Tumor cells were reactive for p53 (1:10,000, Invitrogen), and the Ki-67 labeling index (LI) was $12.2 \%$. Since immunohistochemical findings of the tumor shared characteristics of both RCC and hemangioblastoma, electron microscopy was performed on the paraffin-embedded tumor tissue. Ultrastructurally, the cytoplasm of the tumor cells contained abundant bundles of microfilaments, occasional lipid dro-

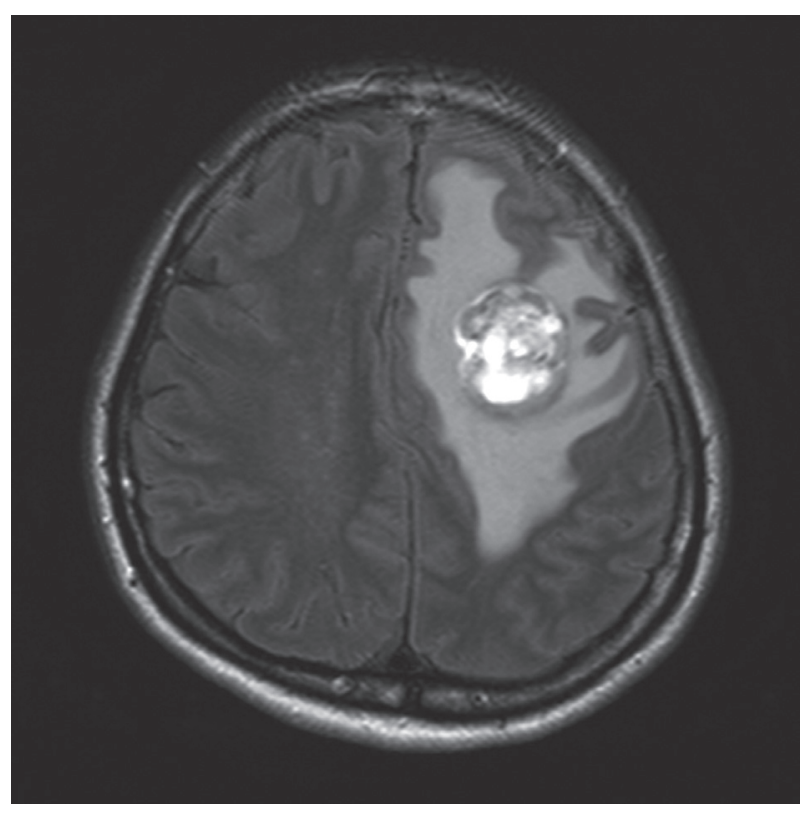

Fig. 1. Brain magnetic resonance imaging (T2 Flair) shows a heterogeneously enhancing mass containing a cystic component in the left frontal lobe. plets, some rough endoplasmic reticulum (RERs), and glycogen particles. The histopathologic, immunohistochemical and ultrastructural findings were compatible with a diagnosis of supratentorial hemangioblastoma. To rule out the possibility of von Hippel-Lindau disease, abdominal computed tomography (CT) was performed along with a family history check. Bilateral kidneys showed up normal on the abdominal CT, and the family history was not significant for any relevant diseases.

\section{DISCUSSION}

A rare case of supratentorial cellular hemangioblastoma with hyaline globules was described. Diagnostic difficulty was experienced in this case because the tumor occurred in a rare site and showed unusual histopathologic findings. The incidence of sporadic supratentorial hemangioblastoma has not yet been described. However, in von Hippel-Lindau disease, the incidence of a supratentorial location has been reported to range from $1 \%-$ $11 \%{ }^{2}$ The unusual histopathologic findings included marked nuclear pleomorphism, uni- and multi-vacuolated cells resembling lipoblasts and abundant hyaline globules. Other unusual findings included expression of CD10 and p53 and a relatively high Ki-67 LI. The stromal cells of hemangioblastoma typically have foamy or vacuolated cytoplasm, which was sparse in our case. The vacuoles were larger than those in typical hemangioblastomas. Hyaline globules are also not common in hemangioblastoma. The Ki-67 LI tends to be low, in the range of $0 \%-$ $2 \%$, but $8.2 \%$ was previously reported in a cellular variant with marked pleomorphism. ${ }^{4}$ The relatively high Ki-67 LI (12.2\%) of this case suggests the diagnosis of a cellular variant of heman-
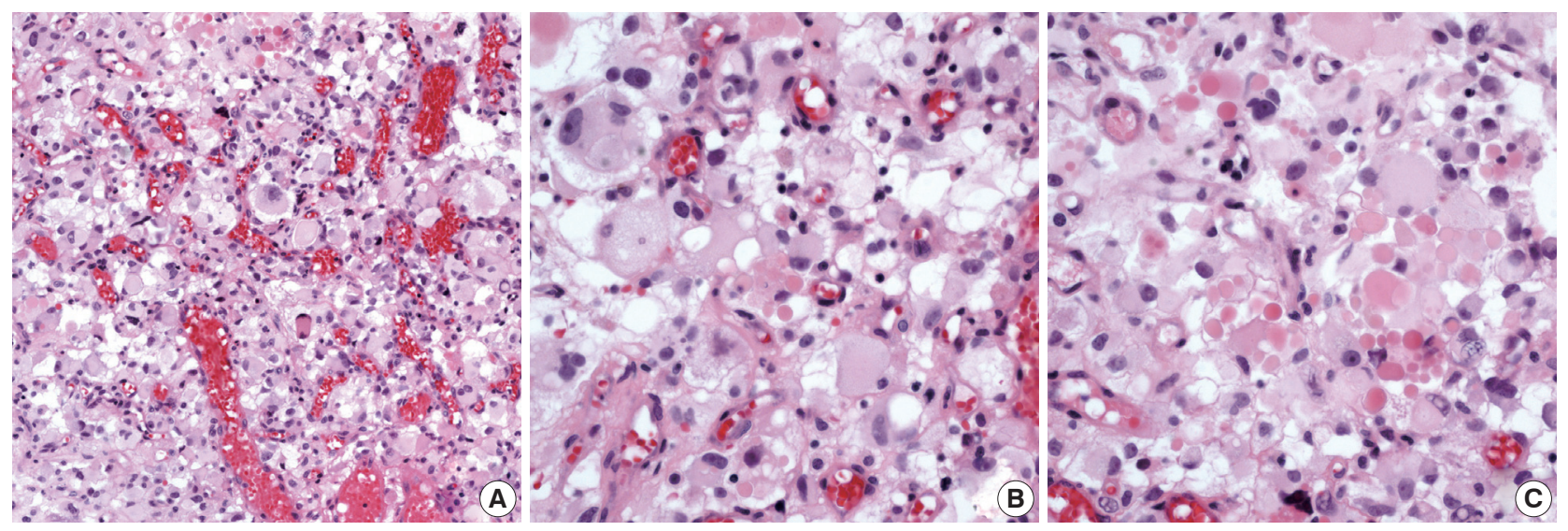

Fig. 2. Histologic features. (A) The tumor consists of sheets of polygonal cells and abundant arborizing capillary networks. (B) The tumor cells show ample and eosinophilic cytoplasm with an occasionally uni- or multi-vacuolated appearance. (C) Numerous extracellular and intracytoplasmic hyaline globules are identified. 

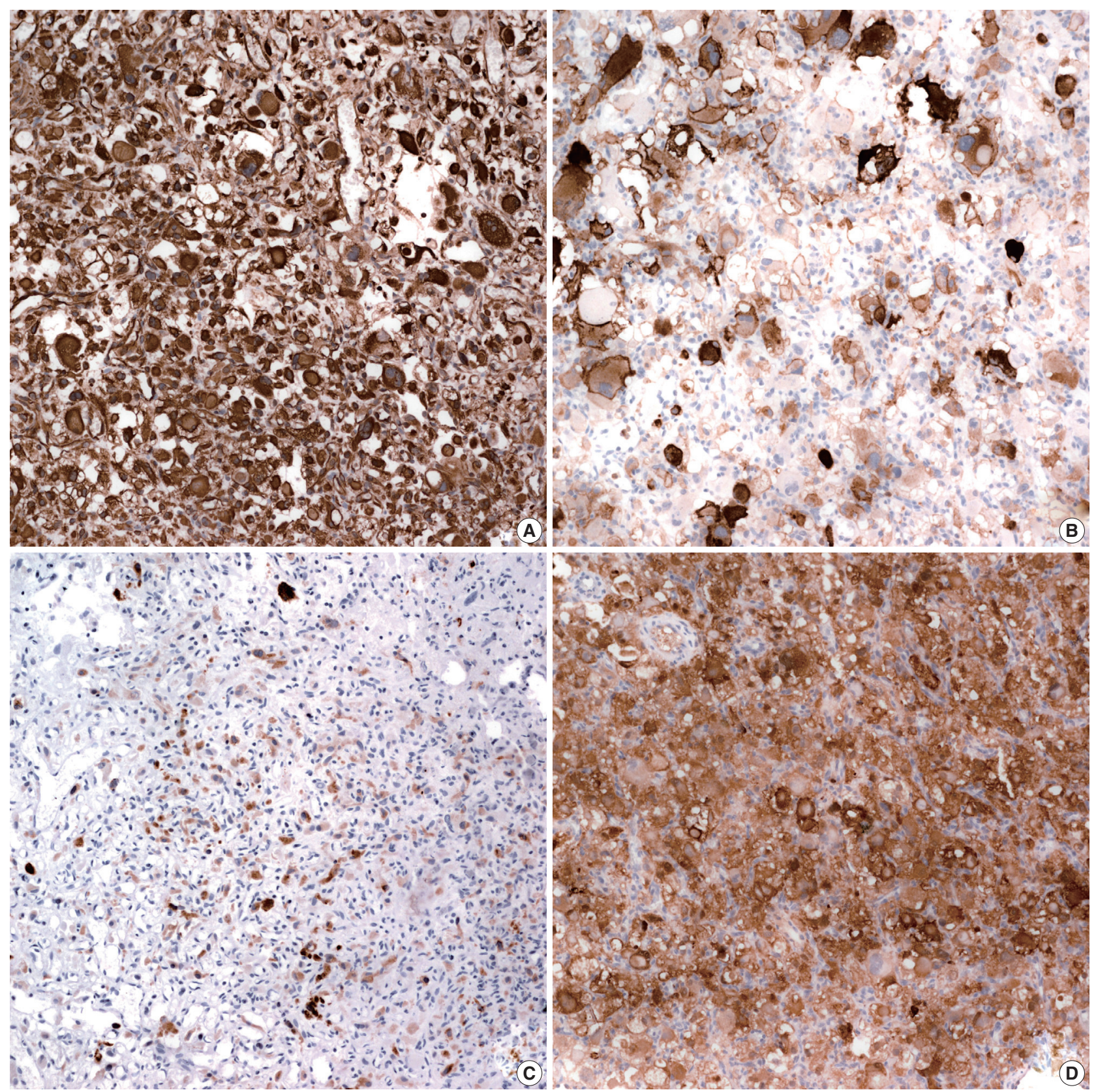

Fig. 3. Immunohistochemical staining. Tumor cells are diffusely positive for vimentin (A) and CD10 (B) and focally positive for a-inhibin (C) and S-100 (D).

gioblastoma. p53 immunostaining has been examined in only six cases of hemangioblastoma, all of which were negative. ${ }^{5}$ The clinical significance of $\mathrm{p} 53$ expression in this case remains unclear.

Distinguishing between hemangioblastoma and metastatic RCC in the CNS based on morphology can be quite difficult. CD10 and $\alpha$-inhibin have been useful markers for the differential diagnosis. The marker $\alpha$-inhibin, which has previously been helpful in the differential diagnosis of RCC from adrenal corti- cal tumors, is a sensitive and specific marker for the stromal cells of hemangioblastoma. The majority of RCC is CD10-positive, whereas hemangioblastomas are negative. However, a recent study by Rivera $e t$ al. ${ }^{6}$ demonstrated a weak positivity for $\mathrm{CD} 10$ in 2 out of 15 hemangioblastomas, consequently proposing the combination of $\alpha$-inhibin and PAX-2 to be the most useful markers to differentiate RCC from hemangioblastoma. RCC shows a positive immunoreaction for PAX-2 but a negative immunoreaction for $\alpha$-inhibin, whereas the immunoreac- 
tivity of hemangioblastoma displays the opposite. The results of immunostaining in the present case ( $\alpha$-inhibin,+ PAX-2 -) are compatible with hemangioblastoma. Other differential diagnoses include metastatic adrenal cortical carcinoma, paraganglioma, pleomorphic round cell tumor and also primary brain tumor. Immunohistochemical studies and histologic findings can help to distinguish hemangioblastoma from these other lesions. Ultrastructurally, the stromal cells of hemangioblastoma share fine features with endothelial cells and pericytes, such as micropinocytic vesicles, lots of microfilaments, hemidesmosomes, basement membranes, and Weibel-Palade bodies. Shimura et al. ${ }^{7}$ reported that most stromal cells of hemangioblastoma have abundant clear cytoplasm containing RER, mitochondria, scattered fibrils, and large lipid inclusions. These ultrastructural features were also identified in our case, in addition to abundant microfilaments.

The origin of the stromal cells of hemangioblastoma is still controversial. In one study, the cells showed no significant staining for endothelial, epithelial, neural, pericytic, or endocrine immunohistochemical markers. ${ }^{8}$ Hyaline globules can be observed in both tumors and benign tissues. Papadimitriou et al. ${ }^{3}$ proposed a model for the formation of hyaline globules in relation to apoptosis. Hyaline globules are more commonly associated with RCC, adrenal cortical carcinoma and paraganglioma than hemangioblastoma. They have been described in a minority of CNS hemangioblastomas ${ }^{9}$ and sporadic renal hemangioblastomas. ${ }^{10}$ In our case, most of the hyaline globules were extracellular, and only a few of the stromal cells had intracellular hyaline globules.

In conclusion, supratentorial hemangioblastoma is a rare benign neoplasm and should be distinguished from various other malignant tumors, which have similar morphologies.

\section{Conflicts of Interest}

No potential conflict of interest relevant to this article was reported.

\section{Acknowledgments}

The authors thank Michael J. Van Vrancken for proofreading.

\section{REFERENCES}

1. Yang B, Luan S, Cao X, Bao W. Supratentorial hemangioblastoma. Neurosciences (Riyadh) 2011; 16: 150-2.

2. Peyre M, David P, Van Effenterre R, et al. Natural history of supratentorial hemangioblastomas in von Hippel-Lindau disease. Neurosurgery 2010; 67: 577-87.

3. Papadimitriou JC, Drachenberg CB, Brenner DS, Newkirk C, Trump BF, Silverberg SG. “Thanatosomes”: a unifying morphogenetic concept for tumor hyaline globules related to apoptosis. Hum Pathol 2000; 31: 1455-65.

4. Ono T, Sasajima T, Oda M, Mizoi K. Cerebellar hemangioblastoma with marked pleomorphism: a case report. No Shinkei Geka 2012; 40: 643-50.

5. Miyagami M, Katayama Y, Nakamura S. Clinicopathological study of vascular endothelial growth factor (VEGF), p53, and proliferative potential in familial von Hippel-Lindau disease and sporadic hemangioblastomas. Brain Tumor Pathol 2000; 17: 111-20.

6. Rivera AL, Takei H, Zhai J, Shen SS, Ro JY, Powell SZ. Useful immunohistochemical markers in differentiating hemangioblastoma versus metastatic renal cell carcinoma. Neuropathology 2010; 30: 580-5.

7. Shimura T, Hirano A, Llena JF. Ultrastructure of cerebellar hemangioblastoma: some new observations on the stromal cells. Acta Neuropathol 1985; 67: 6-12.

8. Frank TS, Trojanowski JQ, Roberts SA, Brooks JJ. A detailed immunohistochemical analysis of cerebellar hemangioblastoma: an undifferentiated mesenchymal tumor. Mod Pathol 1989; 2: 638-51.

9. Burger PC, Scheithauer BW. Tumors of the central nervous system. Washington, DC: American Registry of Pathology in collaboration with the Armed Forces Institute of Pathology, 2007; 310-12.

10. Zhao M, Williamson SR, Yu J, et al. PAX8 expression in sporadic hemangioblastoma of the kidney supports a primary renal cell lineage: implications for differential diagnosis. Hum Pathol 2013; 44: 2247-55. 